\title{
Innovation pédagogique à l'université : comparaison entre apprentissage par problèmes et cours traditionnel
}

Perrine Martin et Philippe Padula

\section{(2) OpenEdition Journals}

Édition électronique

URL : http://journals.openedition.org/ripes/1574

ISSN : 2076-8427

\section{Éditeur}

Association internationale de pédagogie universitaire

\section{Référence électronique}

Perrine Martin et Philippe Padula, « Innovation pédagogique à l'université : comparaison entre apprentissage par problèmes et cours traditionnel », Revue internationale de pédagogie de l'enseignement supérieur [En ligne], 34(3) | 2018, mis en ligne le 20 novembre 2018, consulté le 08 septembre 2020. URL : http://journals.openedition.org/ripes/1574 


\title{
Innovation pédagogique à l'université : comparaison entre apprentissage par problèmes et cours traditionnel
}

\author{
Perrine Martin et Philippe Padula
}

\section{Introduction}

Face à un public massif et divers d'étudiants, la question de la transmission de connaissances dans l'enseignement supérieur et de l'évolution des pratiques pédagogiques pour y répondre est actuellement au cœur des débats (Beaud, 2002; Romainville, 2000). Il s'agit aujourd'hui de penser des méthodes pédagogiques appropriées à un nouveau public demandant une attention particulière (Peretti, 2009). En nous appuyant sur les recherches menées dans le domaine de la pédagogie universitaire, nous savons aujourd'hui que le modèle pédagogique universitaire "traditionnel ", notamment à travers la pratique du cours magistral, ne semble plus correspondre à la demande sociétale (Felouzis, 2003) et au caractère professionnalisant des formations souhaitées par l'université. Sa fonction, historiquement construite, d'élaboration et de transmission orale du savoir (Bruter, 2008) est remise en question. Sa pertinence est critiquée (Berger, 2012). Nous assistons ainsi, au sein des universités, au développement d'une variété d'expérimentations pédagogiques (Clanet, 2001) s'appuyant sur une approche par compétences notamment (Boutin, 2004). Pourtant, ces remises en cause nous interrogent. Le cours magistral existe toujours et nombre d'enseignants universitaires continuent de " parler tout seul devant les étudiants ». La question de l'efficacité de ces expérimentations pédagogiques par rapport à un cours traditionnel se pose donc toujours, ce que nous souhaitons regarder dans cet article dans le cadre de la formation des ingénieurs. 


\section{Formation des ingénieurs à l'université}

Notre étude se situe dans le cadre d'une formation de cinq ans au métier de l'ingénieur, un cursus Master ingénierie (CMI) d'Aix Marseille Université (AMU) en France. Le référentiel des $\mathrm{CMIs}^{1}$ offre une large place aux pédagogies innovantes et impose de réaliser une partie de la formation à travers des activités de mise en situation.

Pour répondre à cette attente, l'usage de l'apprentissage par problèmes (APP) est l'une des préconisations du référentiel CMI. Celle-ci fait écho à une étude de l'Agence d'évaluation de la recherche et de l'enseignement supérieur (2010) qui précise que former un ingénieur ne consiste pas simplement à transmettre des connaissances (permettant le développement de compétences scientifiques et techniques), mais aussi à développer des aptitudes professionnelles (aptitudes personnelles, capacités professionnelles, aptitudes interpersonnelles). Dans ce contexte, Newman (2005) précise que l'APP permet de développer les compétences d'écoute active, de résolution de problèmes, de planification stratégique, de définition de problèmes réels, de prise de décision, ou encore de développement de l'empathie.

\subsection{Enjeux de l'implantation d'un apprentissage par problèmes}

L'implantation d'un enseignement par APP a pour objectif de répondre à trois enjeux principaux :

- Une demande sociétale : la formation au métier de l'ingénieur du XXIe siècle demande de développer des compétences visant la professionnalisation des étudiants (processus de Bologne).

- Une demande institutionnelle préconisée par le référentiel des CMIs (mise en place d'APP dans la formation) : les formations en CMIs, adossées aux laboratoires de recherche, doivent conserver un haut niveau scientifique.

- Un changement pédagogique : il doit être porteur de réussite pour convaincre les acteurs de l'université de changer les habitudes.

Pour autant, la crainte d'une baisse de niveau disciplinaire inquiète une partie du corps professoral. Il s'agit alors de s'interroger sur l'APP en termes d'efficience par rapport à une formation traditionnellement dispensée: en quoi l'APP peut-il favoriser le développement et l'acquisition de connaissances formelles et de compétences chez des étudiants de Licence sciences pour l'ingénieur de manière plus efficace qu'un apprentissage traditionnel?

$6 \quad$ Les résultats de notre étude devraient nous permettre de mettre en lumière les apports et les faiblesses de l'APP en formation d'ingénieur et ainsi de contribuer aux réflexions actuelles sur l'amélioration de la qualité des formations dans l'enseignement supérieur.

\section{Apprentissage par problèmes}

\subsection{Définition}

7 L'APP (Problem-based Learning) est né au milieu des années 1960 à l'Université McMaster en Ontario (Canada) dans le contexte de la formation médicale. C'est une méthode d'apprentissage très structurée par différentes étapes, qui alterne travail en groupe et 
apprentissage individuel. L'APP fait partie des dispositifs d'enseignement qui rompent avec la juxtaposition d'une formation théorique et d'une formation pratique. À l'instar de nombreux auteurs (Savin-Baden, 2000 ; Dochy, Segers, Van Den Bossche et Gijbels, 2003), l'APP est défini comme une approche centrée sur la réactivation des connaissances antérieures et le traitement actif des connaissances par l'étudiant. L'APP s'appuie sur l'intérêt de partir de problèmes signifiants, interdisciplinaires et inspirés de contextes réels pour identifier et construire les ressources cognitives pertinentes, qui seront mobilisées le moment voulu, plutôt que d'accumuler «simplement » des ressources.

8 L'APP s'organise en trois étapes qui se répartissent sur une durée courte (une à deux semaines). Le dispositif de formation classique, défini par Raucent et Milgrom, (2011), est décrit ci-dessous.

- Etape 1 : en groupe. Définition du problème. Le groupe découvre la situation-problème. Il doit s'approprier le problème, faire le bilan des prés-acquis nécessaires et des connaissances à acquérir, définir le plan d'action à mener (ce qui est à rechercher / ce qui est à apprendre). Le groupe est autonome durant cette étape. Les choix stratégiques lui en incombent.

- Etape 2 : apprentissage individuel. Chaque membre du groupe travaille de façon autonome suivant ce qui a été défini au cours de l'étape 1. L'autonomie de recherche d'information est ici privilégiée. Cette étape est au cœur du dispositif d'APP. L'apprenant doit être capable de contrôler son apprentissage, en travaillant des concepts incontournables, et de ne pas se focaliser sur des concepts secondaires, voire hors sujet. La clarification des objectifs lors de l'étape 1 trouve ici tout son sens.

- Etape 3 : en groupe. Cette étape comporte trois temps. A l'aide des nouvelles connaissances dont ils disposent, les membres du groupe mettent en commun les réponses à la situationproblème basées sur la recherche individuelle. Le groupe fait une rétroaction sur son organisation du travail afin d'améliorer son efficacité future. Il analyse les points qui ont bien fonctionnés et ceux qui sont à améliorer. Le but recherché est d'apprendre à travailler en groupe, mais aussi d'améliorer les conditions permettant un apprentissage efficace. Une autoévaluation individuelle, à la fin de l'APP, permet à chaque étudiant de percevoir son degré d'apprentissage et les lacunes à combler.

\subsection{Etat de la connaissance sur l'efficacité de l'APP}

9 En nous intéressant aux différentes études menées sur l'efficacité de l'APP, nous constatons que la grande majorité de celles-ci s'accordent sur le fait que l'APP permet de développer des compétences chez les étudiants. Par exemple, dans le domaine des études médicales, des méta-analyses (Dochy et al., 2003 ; Vernon et Blake, 1993) ont comparé le niveau de connaissances et de compétences des étudiants entre des cursus en APP et des cursus «traditionnels ». Ces études ne font pas apparaître de différences significatives sur des tests de connaissance factuels entre les deux dispositifs. Par contre, en ce qui concerne l'acquisition des compétences, elles tendent à promouvoir les situations d'enseignement organisées autour de l'APP en termes de développement de compétences.

Bédard, Lison, Dalle, Côté et Boutin (2012) se sont intéressé à l'engagement et à la persévérance des étudiants qui évoluent dans formation en APP dans un cursus médical, ainsi qu'en apprentissage par problèmes et par projets en ingénierie (génie 
électrique et génie informatique). Cette étude a montré que l'APP favorise l'engagement et la persévérance des étudiants.

11 Une autre expérimentation, Candis 2000, menée par Galand et Frenay (2005) a retenu particulièrement notre attention. Elle a eu lieu à la Faculté des sciences appliquées de l'Université catholique de Louvain. En 2000, l'UCL a mis en place une innovation (passage de formations traditionnelles à des formations en APP) touchant la formation des étudiants ingénieurs en première et deuxième années. L'UCL a évalué les impacts de ce changement sur les étudiants, mais aussi sur les enseignants. Ainsi, 393 étudiants volontaires (sur les 486 ayant suivi la formation en APP et en « traditionnel ») ont passé en début de troisième année un test standardisé d'une durée de deux heures portant sur quatre points appris pendant leurs deux premières années de formation (résumé d'un texte scientifique anglophone, définition de concepts et estimation d'ordres de grandeur, résolution d'un problème électro-mécanique, résolution d'un problème mathématique). L'expérimentation a consisté à comparer sur deux cohortes d'étudiants (ceux qui ont suivi leur formation en APP et ceux qui l'on suivi de manière «traditionnelle ») les différents résultats au test standardisé. L'expérimentation a, entre autres, montré que les étudiants qui ont suivi un enseignement par APP obtiennent les mêmes résultats en ce qui concernent les connaissances et les définitions. Ils obtiennent par contre de meilleurs résultats pour les explications et les justifications.

12 Le point de vue des étudiants sur le passage à l'APP est intéressant. Ils mettent en avant une meilleure assimilation de la matière, une meilleure discipline de travail, plus d'autonomie, de motivation. Par contre, une minorité a éprouvé de grandes difficultés pour s'adapter aux exigences de l'APP (Dalle et al., 2002).

13 L'interprétation de ces différents résultats montre que l'APP est efficace en ce qui concerne le développement de compétences. Les résultats concernant le développement du niveau des connaissances de haut niveau (qui sont des composantes de la compétence) restent plus mitigés. En fait, les études ne montrent pas de différences significatives, du moins à court terme. Pour Bédard, Turgeon, Tardif et Des Marchais (1995), ces résultats sont probablement plutôt liés à certaines modalités d'évaluation qu'au dispositif d'APP en lui-même.

\subsection{Originalité de la contribution}

Notre recherche vient compléter les études mentionnées précédemment. Elle s'appuie sur un ensemble d'étudiants d'une même formation. Si les expérimentations antérieures se sont intéressées à l'APP sur des formations longues (pour Candis 2000, les étudiants ont suivi leur cursus en APP pendant deux années avant l'évaluation du dispositif), notre étude interroge l'efficacité de l'APP sur l'apprentissage d'étudiants n'ayant pas l'habitude de travailler dans le cadre d'un apprentissage actif. Dans ce contexte, il s'agit de questionner le niveau d'acquisition des procédures, de mise en œuvre de ces procédures et la capacité de résolution de problèmes complexes des étudiants. Les résultats obtenus devraient nous permettre d'aborder la question de l'adaptation nécessaire à toute situation pédagogique nouvelle et, à plus long terme, d'interroger la formation des enseignants universitaires aux pédagogies actives pour mettre en œuvre des situations d'enseignement-apprentissage adaptées. 


\section{Cadre conceptuel}

\subsection{Fondement de l'APP}

15 L'APP s'inscrit dans les courants de la psychologie cognitive et du constructivisme. Il trouve sa source dans les travaux de Dewey qui, au début du XXe siècle, insistait sur le fait que les connaissances ne se transfèrent pas directement et que l'apprentissage requiert une participation active de l'apprenant. Les enseignants sont donc invités à développer une approche pédagogique où des problèmes, tels qu'on peut les rencontrer dans la vie réelle, sont le point de départ de l'apprentissage. Partant de ce principe, l'étudiant se retrouve au centre du processus d'apprentissage, invité à orienter son apprentissage vers une compréhension approfondie des sujets plutôt que de faire appel uniquement à la mémorisation.

\subsection{Notion de situation-problème} situation- problème articule le niveau des représentations et des conceptions des apprenants et le niveau de l'organisation conceptuelle des apprentissages (Cornu et Vergnioux, 1992). Il s'agit ainsi d'organiser précisément l'interaction pour que, dans la résolution du problème, l'apprentissage s'effectue. Pour De Vecchi et CarmonaMagnaldi (2015), la situation-problème est porteuse de sens pour celui qui apprend. En fait, elle renvoie directement à un aspect de la pratique professionnelle et permet de faire le lien entre le monde réel et des connaissances disciplinaires.

En APP, l'apprentissage se réalise à partir de situations authentiques faisant appel à la mobilisation de différentes ressources cognitives que l'apprenant combine pour résoudre la situation-problème. Le travail sur cet obstacle épistémique s'appuie alors sur des conflits cognitifs ou sociocognitifs et son dépassement ouvre sur la construction de connaissances à caractère général. L'apprentissage à l'œuvre dans ce type de situation relève de travaux développés par Piaget. En effet, le découpage et l'emboitement des situations assurant sa progression permet aux mécanismes d'accommodation-assimilation de fonctionner et amène ainsi les étudiants à progresser dans l'appropriation des connaissances, et ce, en situation d'intégration.

\subsection{Notion de compétence dans le champ de l'éducation}

Comme mentionné en introduction, le modèle d'un enseignement transmissif favorisant l'apprentissage de comportements types, apparaît peu opératoire à l'université. A contrario, le modèle de la compétence, conçue comme une organisation cognitive évolutive permettant d'apporter des réponses comportementales adaptées en fonction des caractéristiques d'une situation, semble plus pertinent.

En éducation, la référence aux compétences a émergé des théories cognitives, qui s'intéressent au traitement de l'information opéré par le sujet dans la conduite de son activité. L'arrivée de la notion de compétence témoigne du passage d'une référence béhavioriste à une référence cognitiviste. Il ne s'agit plus de faire produire des

Revue internationale de pédagogie de l'enseignement supérieur, 34(3) | 2018 
comportements reproductibles, mais de développer des dispositions à générer des conduites adaptées face à des situations diverses et changeantes.

En dépit des très nombreuses définitions de la compétence, il y a un assez large consensus pour la considérer comme une capacité d'exécution finalisée, liée à l'activité située d'un sujet et correspondant chez lui à une organisation d'éléments divers, notamment des savoirs et des savoir-faire, ou d'unités cognitives construites, coordonnées, intégrées, structurées, hiérarchisées (Aubret, Gilbert et Pigeyre, 1993; Bellier, 1999; Leplat, 2000). Ainsi, la compétence pourrait se définir comme la mobilisation intériorisée et réfléchie d'un ensemble de ressources pour faire face à des situations complexes (Roegiers, 2014). Dans le champ de l'éducation, une personne est donc considérée compétente si elle peut mettre en œuvre de façon cohérente un certain nombre de ressources et agir efficacement dans une famille de situations et un contexte déterminé (Perrenoud, 1997; Tardif, 2003). Entendons par ressources, un ensemble plus ou moins importants de ressources cognitives (internes à l'individu) et non cognitives (externes à l'individu), dépendant autant de la situation et du contexte que de la personne elle-même et des actions qu'elle est disposée à mener dans cette situation. Rey, Carette, Defrance et Kahn (2012) précisent qu'une compétence est toujours définie par la tâche et non pas par des processus psychologiques ou physiologiques qui doivent être activés dans l'exercice de la compétence. Il est essentiel d'établir une distinction entre la tâche, c'est-à-dire le but à atteindre, qui est une activité finalisée (Rey et al., 2012), et les conditions dans lesquelles elle doit être réalisée, et l'activité, à savoir «ce qui est mis en œuvre par le sujet pour exécuter la tâche » (Leplat, 2004, p. 102). Quant à la situation, elle peut être définie comme tous les éléments contextuels susceptibles d'affecter l'interaction du sujet avec la tâche (Chauvigné et Coulet, 2010). La figure 1 modélise la définition de la compétence que nous retenons. 
Figure 1. Définition retenue de la compétence

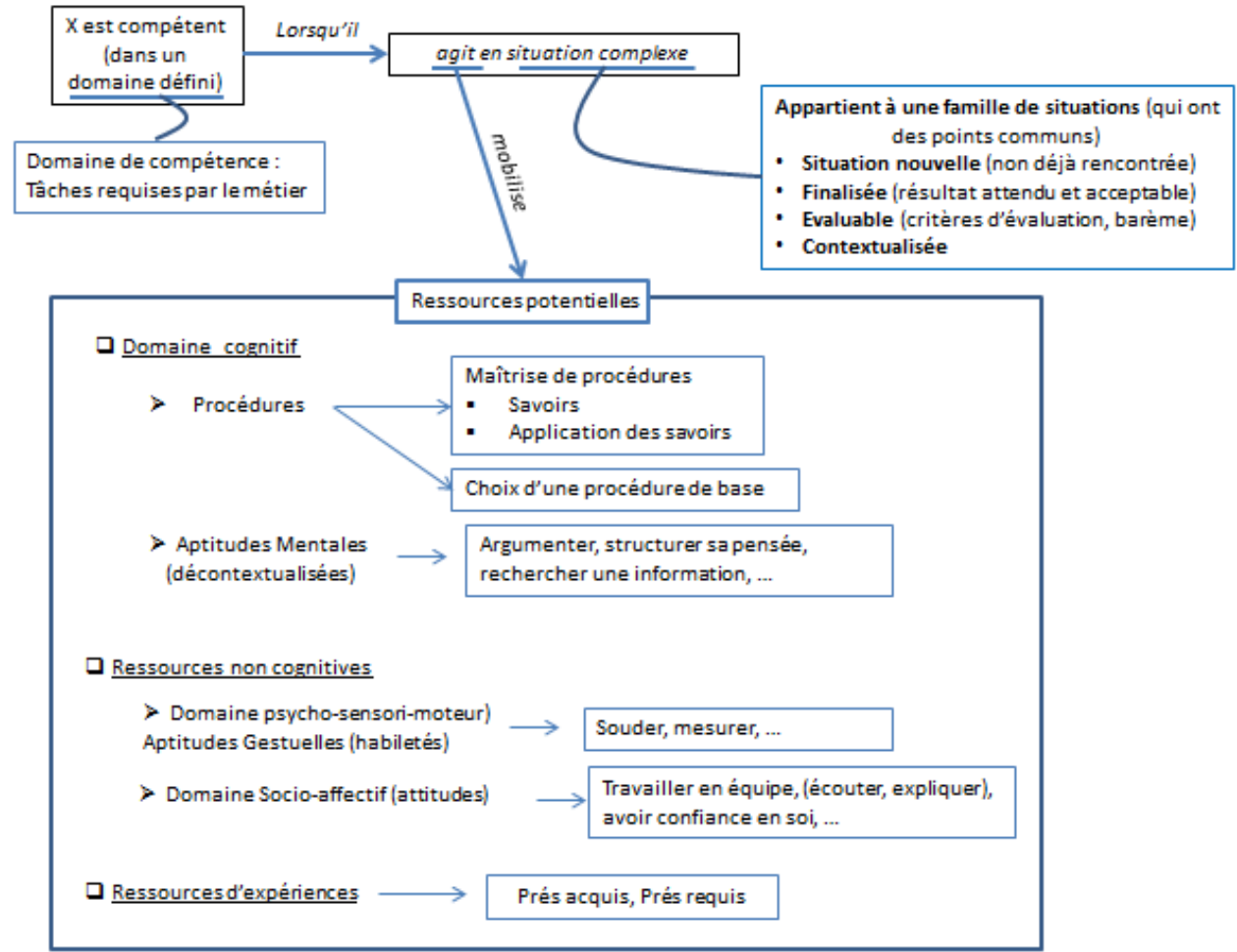

\section{Question et hypothèses de recherche}

Notre question de recherche est la suivante: mettre en place l'APP est-il efficace au niveau de l'acquisition de compétences des étudiants? Rey et al. (2012) proposent un modèle de l'évaluation de la compétence en trois degrés :

- Premier degré : La procédure ou compétence élémentaire (encore dénommée habileté par les anglo-saxons) : il s'agit de savoir exécuter une opération (ou une suite prédéterminée d'opérations) en réponse à un signal préétabli, après entrainement.

- Deuxième degré: La compétence élémentaire avec cadrage: il s'agit de choisir la compétence élémentaire qui convient (dans l'ensemble des compétences élémentaires que l'on connait) à une situation inédite qu'il est nécessaire d'interpréter.

- Troisième degré: La compétence complexe: il s'agit de savoir choisir et combiner correctement plusieurs compétences élémentaires pour traiter une situation nouvelle et complexe.

Nous faisons ainsi l'hypothèse que l'enseignement dispensé en APP va permettre aux étudiants d'acquérir de manière efficace des procédures (savoirs et applications des savoirs et résolution de problèmes guidés) et de mettre en œuvre ces procédures lors de la résolution d'un problème complexe, donc d'acquérir ces compétences.

Enfin, les modalités d'organisation de l'APP s'appuient sur l'autonomie de l'apprenant et sur une différentiation pédagogique dans les apprentissages permettant ainsi aux étudiants d'apprendre en fonction de leurs besoins. Cette autonomie implique l'engagement des étudiants. Cette modalité est-elle favorable à tous les étudiants quel que soit leur niveau initial? 


\section{Méthodologie}

\subsection{Contexte et participants}

24 Notre étude s'appuie sur la comparaison de deux situations d'enseignement, l'une traditionnelle (cours magistral (CM) et travaux dirigés (TD)) et l'autre en APP, en deuxième année de Licence sciences pour l'ingénieur, au semestre quatre, pour l'unité d'enseignement Etude des systèmes mécaniques. Dans les deux situations de formation, les enseignants sont expérimentés. L'expérimentation a eu lieu chronologiquement sur trois contenus d'apprentissage: la cotation fonctionnelle (thème 1); le guidage en rotation par contact radial (thème 2 ); le guidage en rotation par contact oblique (thème 3).

25 Compte tenu, du faible nombre d'étudiants, l'expérimentation a été menée sur deux promotions d'étudiants, en 2014 et 2015. Au total, le groupe APP était constitué de 35 étudiants et le groupe CM/TD traditionnel de 24 étudiants. Mentionnons que les étudiants du groupe APP n'avaient jamais travaillé en APP. Par ailleurs, une analyse préalable (voir annexe 1) a montré que les deux promotions obtenaient en moyenne des résultats similaires.

\subsection{Expérimentation}

L'unité d'enseignement Etude des systèmes mécaniques dans laquelle l'expérimentation s'est déroulée d'une durée de 60 heures (4 heures consécutives chaque semaine pendant 15 semaines). Le curriculum définit les modalités pédagogiques sous forme de $\mathrm{CM}$ et de TD en présentiel. L'insertion de l'APP dans le curriculum existant est décrite en annexe 2 .

Ceci nous a amenés à proposer trois recueils de données quantitatives qui s'inscrivent dans la phase d'enseignement et d'apprentissage, sous forme de tests (pré-test, posttest, test intégrateur). Ces tests répondent à un cahier des charges que nous définissons ci-dessous.

- Pour être compétent, l'étudiant doit maîtriser les savoirs et leurs applications (degrés 1 et 2 du modèle d'évaluation de la compétence développé par Rey et al. (2012)).

- Pour vérifier la progression, il nous faut connaître en amont de la formation le niveau de connaissance des étudiants, certains ayant ont des pré-acquis disciplinaires.

- La compétence doit s'évaluer dans l'action de réalisation d'une tâche (degré 3 du modèle d'évaluation de la compétence développé par Rey et al. (2012)).

\subsubsection{Pré-test}

$\mathrm{Au}$ regard de leur origine disciplinaire diverse (baccalauréat technique, scientifique Science et vie de la terre ou Science de l'ingénieur), les compétences des étudiants acquises dans leur cursus antérieur sont variées. Pour certains, les trois contenus d'apprentissage ou une partie de ceux-ci pouvaient avoir déjà été abordés. Pour comprendre l'apport réel de notre dispositif de formation, la prise en compte du niveau de connaissances antérieures des étudiants était donc indispensable. Pour ce faire, en amont de la formation, tous les étudiants ont été évalués individuellement par un prétest sur chacun des trois contenus de formation à l'aide de trois exercices indépendants et sans avoir recours à des ressources. Le premier exercice consistait à mesurer le 
niveau d'acquisition des savoirs (connaissances des règles, des définitions, des concepts). Le deuxième exercice mesurait le niveau d'application des savoirs. Le troisième exercice consistait en un problème contextualisé pour lequel les étudiants devaient choisir une procédure pour résoudre le problème à travers une série de questions.

\subsubsection{Test : phase d'enseignement et d'apprentissage}

Pour la phase de test, les séances d'enseignement-apprentissage ont été suivies par deux groupes d'étudiants, l'un CM/TD traditionnel et l'autre APP. Les mêmes contenus disciplinaires, sur les mêmes semaines, ont été travaillés dans les deux groupes. L'enseignant du groupe CM/TD traditionnel a dispensé le cours, puis les étudiants ont appliqué les nouvelles connaissances au travers d'exercices variés et de difficultés croissantes. L'enseignant du groupe APP a mis en place la démarche d'APP telle que développée précédemment.

\subsubsection{Post-test}

30 A la suite de chaque séance d'enseignement-apprentissage les étudiants ont été évalués (post-test). L'objectif étant de mesurer le degré d'acquisition des procédures (maîtrise des procédures - savoirs, applications des savoirs et choix d'une procédure de base) et de comparer la progression d'apprentissage (gain relatif) en fonction des séances d'enseignement-apprentissage (traditionnelles ou APP). Pour nous permettre cette comparaison, le post-test a été identique au pré-test (individuellement et sans ressource).

\subsubsection{Test intégrateur}

31 A l'issue des trois contenus d'apprentissage, les étudiants ont été soumis à un test intégrateur individuel d'une durée de deux heures. Les étudiants étaient mis en conditions professionnelles. Le test intégrateur avait pour objectif de nous informer sur le niveau de compétence des étudiants (degré 3 définit par Rey et al. (2012)) lors de la résolution d'un problème demandant la mobilisation des connaissances. Ainsi, à partir d'un cahier des charges, ils devaient prendre des décisions, fournir une réponse socialement acceptable et justifier leurs choix, et ce, en ayant accès à tous leurs documents. Notons que le résultat au test intégrateur n'a été pris en compte dans la moyenne de l'unité d'enseignement qu'en cas de réussite, pouvant alors être perçu comme un bonus.

La figure 2 reprend la chronologie de l'expérimentation et fait apparaître la progression attendue dans l'acquisition des compétences. 
Figure 2. Chronologie de l'expérimentation

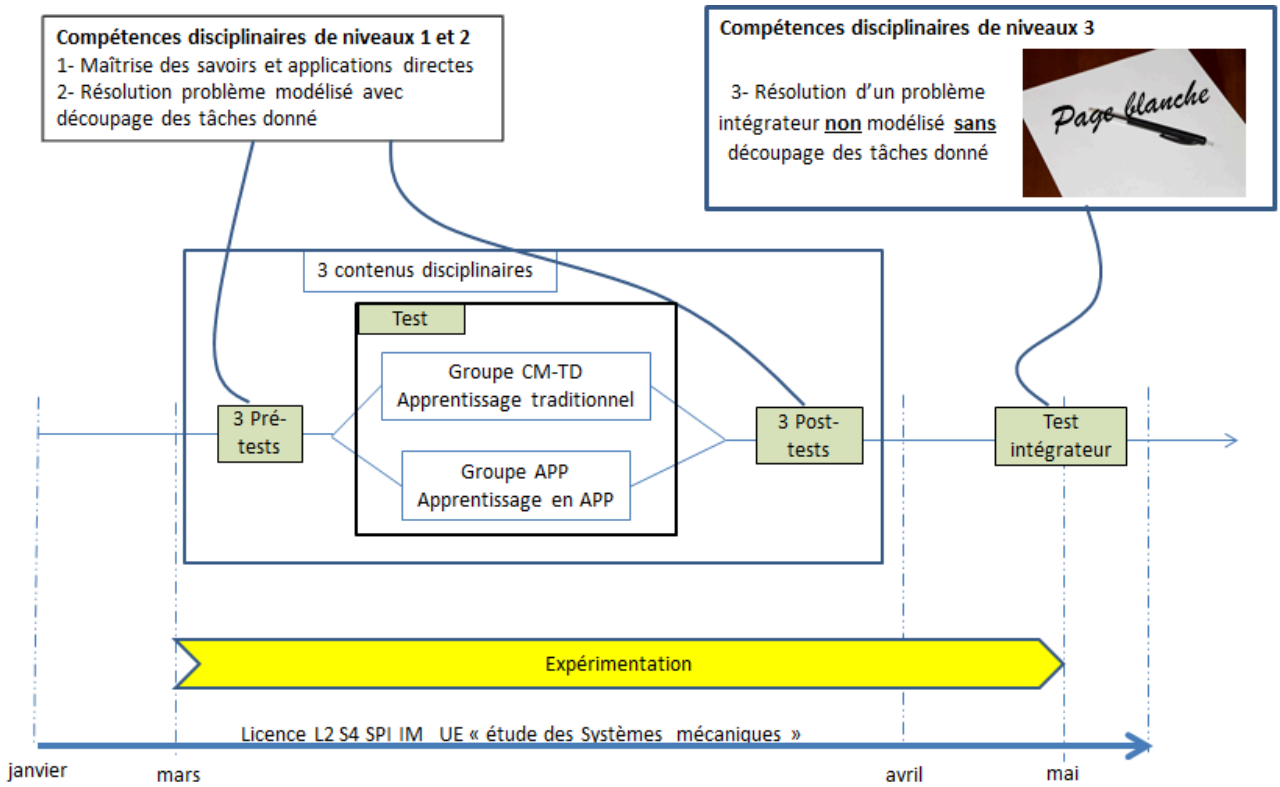

\subsection{Recueil des données}

Les données recueillies ont été analysées de façon mono-variée ou bi-variée à l'aide d'Excel. Pour comparer les deux situations d'enseignement-apprentissage, les données recueillies ont été analysées en fonction de certains critères d'efficacité définis par les travaux de Gérard (2003). Nous retiendrons le niveau d'acquisition qui nous permet de voir si les objectifs de formation sont atteints efficacement et la mise en œuvre des savoirs au travers de la résolution d'un problème complexe.

\subsubsection{Acquisition des procédures}

L'apprenant devrait avoir un score au post-test supérieur ou égal à un indice cible (Ic) défini dans le curriculum de la formation. Les scores des tests sont exprimés sur une échelle variant de 0 à 1,1 correspondant à $100 \%$ de réussite. Nous avons fait le choix de classer les scores en quatre catégories :

- Scores dans la plage $[0-0,35]$, correspondant à une très faible acquisition ;

- Scores dans la plage [0,36 - 0,5], correspondant à une faible acquisition ;

Pour les étudiants qui sont positionnés sur ces deux plages, nous considérons que la formation n'a pas été efficace en termes de résultats.

- Scores dans la plage. [0,5 - 0,65], correspondant à une bonne acquisition ;

- Scores dans la plage. [0,66 - 1], correspondant à une très bonne acquisition.

Le choix de définir la réussite à partir de l'indice cible $(\mathrm{Ic}=0,5)$ correspond aux critères de réussites définis dans le curriculum. 


\subsubsection{Progression}

37 Le gain relatif ${ }^{2}$ 'apprentissage (GR) du dispositif de formation doit être le plus grand possible, mettant ainsi en évidence la progression des étudiants. À nouveau, nous avons fait le choix de classer les gains relatifs en quatre catégories :

- GR dans la plage [0 - 0,35], correspondant à une très faible progression ;

- GR dans la plage [0,36 - 0,5], correspondant à une faible progression ;

Pour les étudiants qui sont positionnés sur ces deux plages, nous considérons que la formation n'a pas été efficace en termes de progression; Gérard (2003) définissant qu'une formation technique est efficace si GR $>0,5$.

- GR dans la plage $[0,5-0,65]$, correspondant à une bonne progression ;

•GR dans la plage [0,66 - 1], correspondant à une très bonne progression.

\subsubsection{Mise en œuvre} problème complexe. L'indicateur de réussite retenu pour cet item consiste à évaluer la compétence de résolution d'un problème complexe sur le test intégrateur qui mobilise des connaissances et des aptitudes de recherche d'information, travaillées sur les trois contenus d'apprentissage.

Pour analyser les données relatives à l'acquisition de compétences en termes de mise en œuvre, les scores de chaque étudiant obtenus au test intégrateur ont été classés en quatre catégories :

- Scores dans la plage [0 - 0,35], correspondant à une très faible mise en œuvre.

- Scores dans la plage [0,36 - 0,5], correspondant à une faible mise en œuvre.

Pour les étudiants qui sont positionnés sur ces deux plages, nous considérons que la formation n'a pas été efficace en termes de mise en œuvre des ressources dans la résolution de problème complexe.

- Scores dans la plage [0,5 - 0,65], correspondant à une bonne mise en œuvre.

-Scores dans la plage [0,66 - 1], correspondant à une très bonne mise en œuvre.

\subsubsection{Synthèse}

La figure 3 ci-dessous modélise le recueil des données quantitatives ainsi que les indicateurs de mesure. 
Figure 3. Recueil des données quantitatives

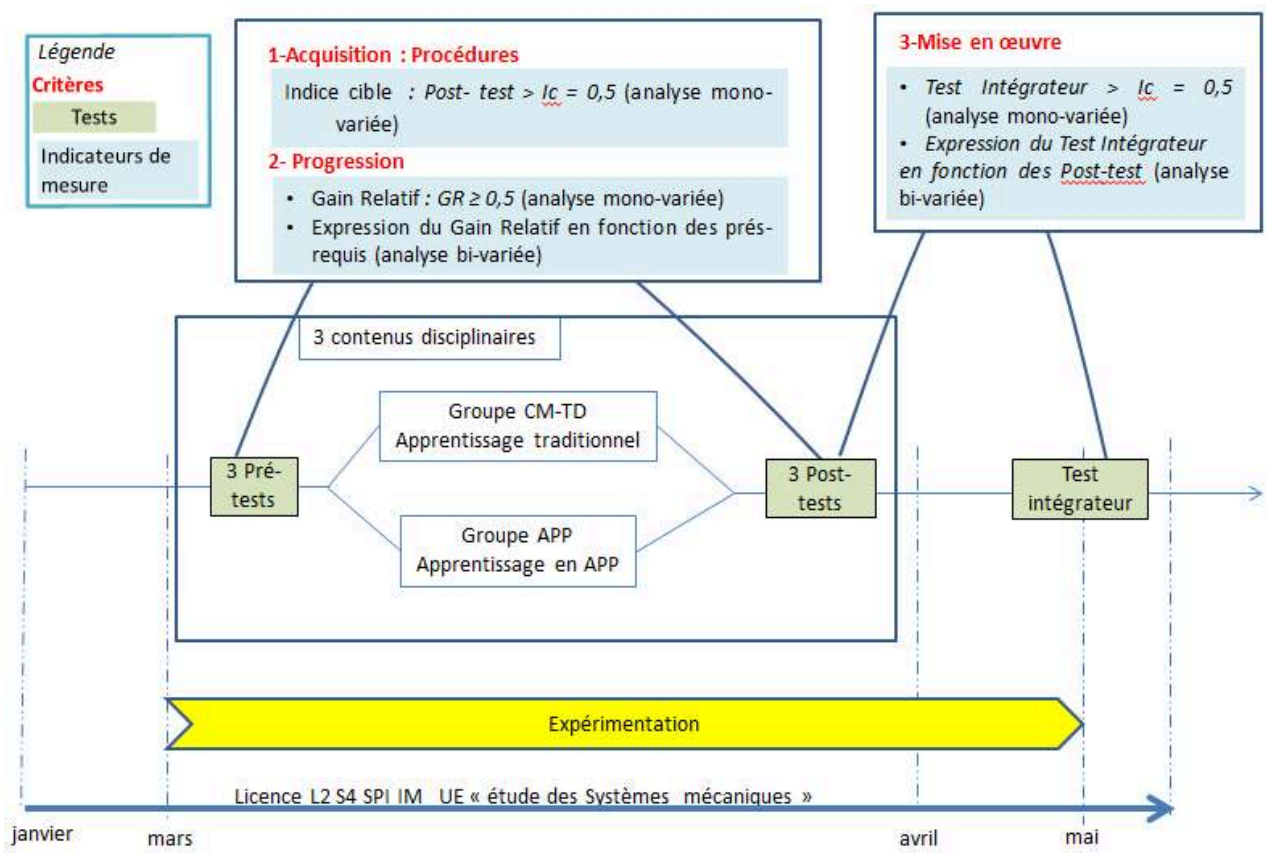

\section{Résultats}

\subsection{Acquisition des procédures}

En ce qui concerne l'acquisition de procédures, la figure 4 détaille de manière descendante l'acquisition des procédures (scores aux post-tests). Celle-ci se scinde en deux: la maîtrise des procédures et le choix d'une procédure. La maîtrise des procédures correspond à la maîtrise et l'application des savoirs.

Figure 4. Acquisition des procédures

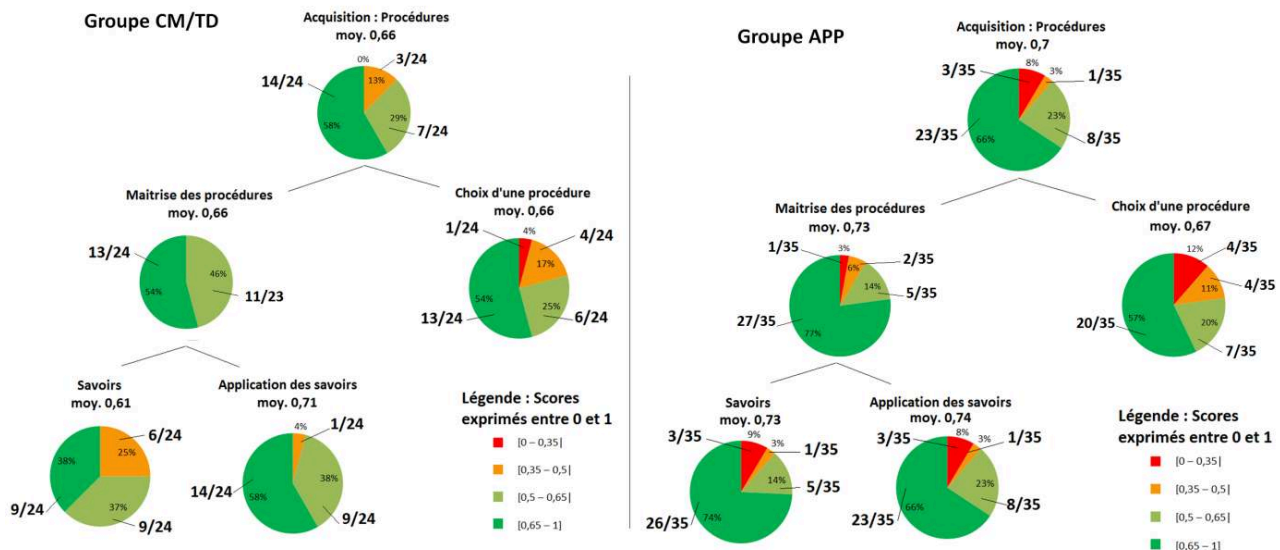

Nos résultats montrent que la moyenne générale est légèrement supérieure pour le groupe APP $(0,70)$ par rapport au groupe CM/TD traditionnelle $(0,66)$. Bien qu'ils ne soient pas statistiquement significatifs, ces résultats ouvrent des pistes de réflexions 
intéressantes. Nous présentons ces résultats plus en détail dans les paragraphes qui suivent.

Lorsque nous entrons dans le détail concernant la maitrise des procédures, nous constatons que le groupe APP maitrise mieux les savoirs en moyenne ( 0,73 contre 0,66 pour le groupe CM/TD traditionnel). Pour ce qui est de l'application des savoirs, les résultats en termes de réussite semblent légèrement supérieurs pour le groupe $\mathrm{CM} / \mathrm{TD}$ traditionnel : 23 étudiants sur 24 (96\%) dépassent l'indice cible alors que 31 étudiants sur $38(89 \%)$ du groupe APP le dépassent. Concernant le choix des procédures, la moyenne générale ne varie pas entre les deux groupes : 0,66 pour le groupe $\mathrm{CM} / \mathrm{TD}$ traditionnel et de 0,67 pour le groupe APP.

La réussite est quasiment identique entre les deux groupes : 19 étudiants sur 24 (79\%) pour le groupe CM/TD traditionnel contre 27 étudiants sur 35 (77\%) pour le groupe APP. La différence n'apparaitt pas dans la tranche supérieure : $57 \%$ pour le groupe APP contre $54 \%$ pour le groupe CM/TD traditionnel ont un score $\geq 0,65$. Par contre, elle semble plus importante au niveau inférieur : $12 \%$ pour le groupe APP contre $4 \%$ pour le groupe $\mathrm{CM} / \mathrm{TD}$ traditionnel ont un score $<0,35$. Les deux groupes atteignent les compétences de degré 1 et 2 nécessaires à la mobilisation dans la résolution d'un problème nouveau.

\subsection{Progression}

Dans un premier temps, nous avons calculé le GR pour chacun des tests individuellement, puis fait la moyenne des trois GR entre les pré-tests et les post-tests pour chaque étudiant. La figure 5 présente la progression du niveau d'acquisition. Celle-ci se scinde en deux, à savoir les GR sur la maîtrise des procédures et sur le choix d'une procédure.

Figure 5. Progression du niveau d'acquisition

\section{Groupe CM/TD}

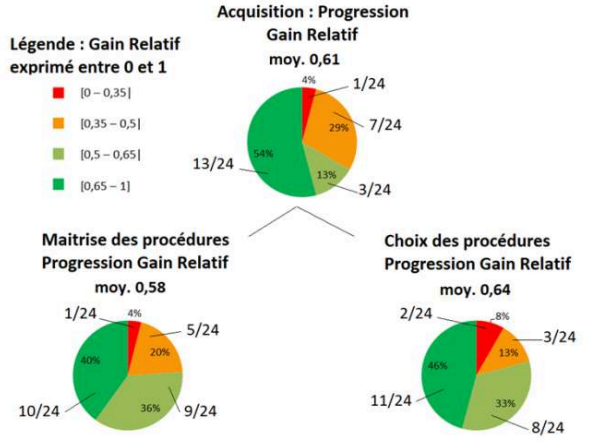

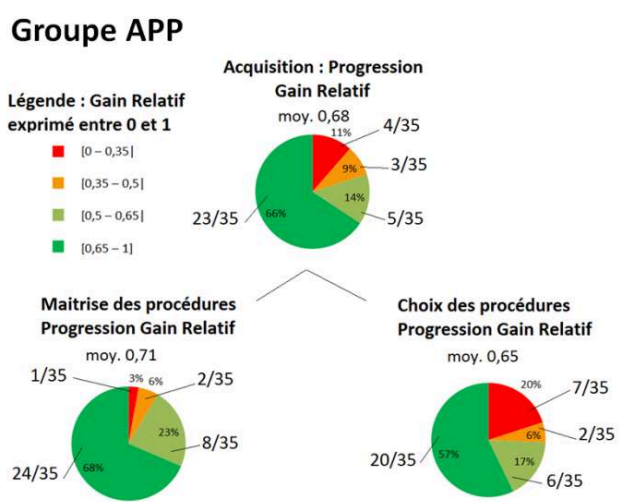

Les résultats montrent que la moyenne générale de progression est légèrement supérieure pour le groupe APP (0,68 contre 0,61 pour le groupe CM/TD). Nous retrouvons la même tendance que précédemment, à savoir une progression pour les deux groupes $(\mathrm{GR} \geq 0,5)$, avec tout de même un avantage pour le groupe APP : 28 étudiants sur $35(80 \%)$ du groupe APP ont une bonne ou très bonne progression contre 16 étudiants sur 24 (67\%) du groupe CM/TD. Les scores du groupe APP sont un peu plus hétérogènes : 23 étudiants sur $35(66 \%)$ du groupe APP progressent très bien contre 13 
étudiants sur 24 (54\%) pour le groupe CM/TD ; 4 étudiants sur 35 (11\%) du groupe APP ont une progression très faible contre 1 étudiant sur 24 du groupe CM/TD.

De manière plus fine, en ce qui concerne la maîtrise des procédures, la moyenne générale de progression est supérieure pour le groupe APP ( 0,71 contre 0,58 pour le groupe $\mathrm{CM} / \mathrm{TD}$ ). Bien qu'il importe de mentionner que nos résultats ne sont pas statistiquement significatifs, ils nous permettent d'envisager des premiers éléments de réponses, notamment en ce qui concerne la progression avec 32 étudiants sur 35 (91\%) du groupe APP qui ont une bonne ou une très bonne progression contre 19 étudiants sur 24 (76\%) du groupe CM/TD.

En ce qui concerne le choix des procédures, la moyenne générale de progression est quasiment identique pour les deux groupes ( 0,64 pour la groupe CM/TD et 0,65 pour le groupe APP) : 19 étudiants sur 24 (79\%) du groupe CM/TD ont une bonne ou très bonne progression contre 26 étudiants sur 35 (74\%) du groupe APP.

\section{3. Étude par niveau d'entrée}

51 Au regard des pré-acquis des étudiants en amont de la formation, il est intéressant de savoir si tous ont pu tirer parti au mieux de leur formation. La formation proposée en APP est-elle adaptée à l'hétérogénéité des étudiants en début de formation ? La figure 6 présente le gain relatif en fonction du niveau initial des étudiants.

Figure 6. Expression du GR en fonction du niveau d'entrée (pré-tests)

Groupe CM/TD

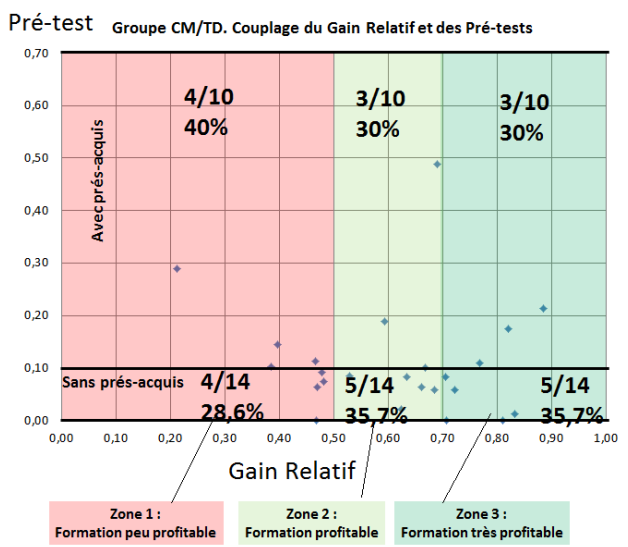

Groupe APP

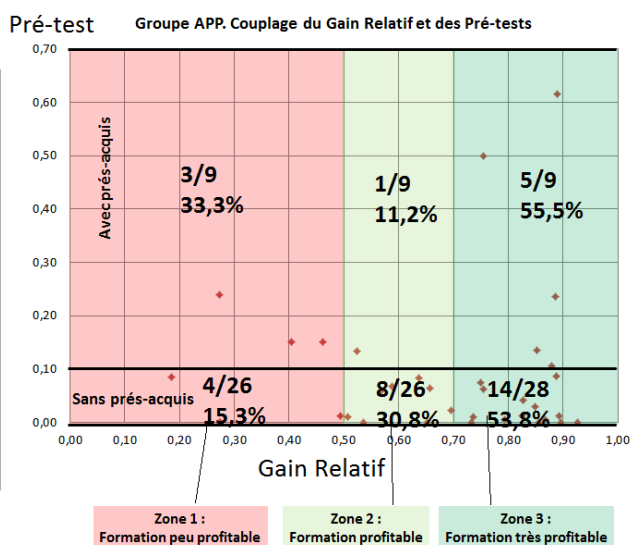

Les données recueillies nous permettent d'analyser les résultats obtenus par les étudiants qui n'avaient pas ou peu de prés-acquis (Score pré-tests $<0,1$ ). Pour le groupe $\mathrm{CM} / \mathrm{TD}, 4$ étudiants sur $14(28,6 \%)$ qui n'avaient pas de prés-acquis ont eu un $\mathrm{GR}<0,5$; 5 étudiants sur $14(35,7 \%)$ un GR compris entre 0,5 et 0,7 et 5 étudiants sur 14 (35,7\%) un GR > 0,7. Pour le groupe APP, 4 étudiants sur $26(15,3 \%)$ qui n'avaient pas de présacquis ont eu un GR $<0,5 ; 8$ étudiants sur $26(30,8 \%)$ un GR compris entre 0,5 et 0,7 et 14 étudiants sur 28 (53,8\%) un GR > 0,7. Dans ce contexte, et bien que nos résultats ne soient pas statistiquement significatifs, il semblerait que l'APP ait permis aux étudiants sans prés-acquis de progresser davantage que ceux du groupe CM/TD.

Cette tendance se retrouve pour les étudiants qui avaient des prés-acquis (Score prétests $\geq 0,1)$. Pour le groupe CM/TD, 4 étudiants sur 10 (40\%) ont eu un $\mathrm{GR}<0,5 ; 3$ étudiants sur 10 (30\%) un GR compris entre 0,5 et 0,7 et 3 étudiants sur 10 (30\%) un GR 
$>0,7$. Pour le groupe APP, 3 étudiants sur $9(33,3 \%)$ ont eu un GR $<0,5 ; 1$ étudiant sur 9 $(11,2 \%)$ un GR compris entre 0,5 et 0,7 et 5 étudiants sur $9(55,5 \%)$ un GR $>0,7$.

\section{Discussion et conclusion}

Notre interrogation était de savoir si la formation en APP permettait aux étudiants d'acquérir les procédures (maitrise des procédures et choix de procédures dans la résolution d'un problème guidé). Comme nous venons de le montrer à travers nos résultats, nous constatons que le groupe APP semble mieux maîtriser les procédures ( 0.73 vs 0.66$)$ et notamment que la moyenne générale de progression est supérieure pour le groupe APP ( 0.68 vs 0.61$)$. Même si nous avons conscience que statistiquement ces résultats ne sont pas significatifs, dans le contexte d'apprentissage étudié, il semble quand même que l'APP ait permis aux étudiants d'atteindre un "bon niveau " disciplinaire. Ces résultats font échos aux différents travaux ayant mis en avant que l'APP était favorable à la compréhension des contenus étudiés et au développement de la capacité à appliquer de nouvelles connaissances (Dochy et al., 2003). L'hypothèse selon laquelle l'engagement des étudiants en APP leur permet de mieux progresser qu'en formation traditionnelle semblerait se confirmer et mériterait une étude complémentaire pour vérifier de manière statistiquement significative les tendances observées dans nos résultats. En ce sens, l'APP permettrait une meilleure progression d'ensemble que la formation «traditionnelle». Rappelons, dans ce sens, que plusieurs études ont démontré que l'APP favorise la pensée réflexive, la communication en groupe (Pastirik, 2006), la résolution de problèmes, l'autorégulation et la collaboration (Hmelo-Silver, 2004).

Nos résultats laissent à penser que l'APP pourrait permettre aux étudiants sans présacquis de mieux progresser par rapport à ceux du groupe CM/TD. Le dispositif en APP semblerait permettre à un groupe hétérogène d'acquérir des compétences au regard de son niveau initial. L'APP, faisant appel à des modalités d'apprentissage basées sur l'autonomie et l'autoévaluation de l'apprenant, permet à chacun de consacrer son temps de travail en fonction de ses besoins. Nos résultats se rapprochent ici des études qui tendent à affirmer l'avantage de l'APP sur l'enseignement traditionnel en termes de développement de connaissances et de compétences (Dochy et al., 2003).

L'APP semble ainsi favorable à la progression des étudiants indépendamment de leur niveau d'entrée avant formation. Le contexte dans lequel l'APP s'opère permet à chacun des étudiants de consacrer son temps et de choisir les contenus d'apprentissage en fonction de ses besoins. En CM/TD, les étudiants doivent suivre le cours au rythme imposé par l'enseignant. Ce rythme peut s'avérer trop rapide ou trop lent pour certains d'entre eux. Dans tous les cas, il ne peut satisfaire pleinement l'hétérogénéité d'une classe. Lors des TD, les étudiants disposent d'un peu plus d'autonomie, mais restent fortement guidés par un panel d'exercices imposés. Les résultats sur la progression concordent avec ce qu'annoncent Derry, Levin, Osana, Jones et Peterson (2000), à savoir que dans une approche d'APP les étudiants obtenaient de bons gains sur les apprentissages dans l'ensemble, mais que ces gains étaient hétérogènes.

En conclusion, notre expérimentation va dans le même sens que nombreuses recherches mettant en avant l'efficacité d'un enseignement par APP en ce qui concerne le développement de compétences. Le travail que nous avons présenté dans cet article avait pour objectif de comparer, un enseignement dispensé de façon " traditionnelle » 
et une démarche d'APP. Nos résultats, qui ne peuvent être généralisés, notamment à cause des effectifs relativement faibles, tendent à montrer que le dispositif d'APP est efficace pour des étudiants qui n'ont pas l'habitude des apprentissages actifs.

La crainte que le développement des aptitudes ne se fasse au détriment des compétences disciplinaires ne semble pas fondée dans notre expérimentation. Le nouveau rapport à l'apprentissage et l'autonomie n'ont pas été défavorables pour les résultats des étudiants. Nous retenons par contre que la question de la place du tutorat dans l'APP est sans doute à explorer.

Par ailleurs, nous avons bâti notre expérimentation sur des recueils de données quantitatives et nous avons conscience que les données obtenues dans cette première recherche ne sont pas suffisantes pour conclure aux effets du dispositif APP sur la réussite (ou l'échec) aux tests. Il s'agira donc de poursuivre ce travail sur un nombre plus important d'étudiants et d'ouvrir le champ des variables à interroger.

Nous retenons également que, dans le cadre de l'APP, le rôle de l'enseignant évolue. Il serait donc pertinent de mener une étude en sollicitant les outils d'analyse du travail pour développer et connaître le travail enseignant. Par exemple, il serait pertinent de recueillir des données d'observation de l'activité au moyen de traces audiovisuelles (Mollo et Falzon, 2004), enrichies par les verbalisations a posteriori des enseignants, afin de comprendre les compromis entre les normes personnelles de viabilité et les normes prescrites par l'institution (Ria, 2009).

\section{BIBLIOGRAPHIE}

Agence d'évaluation de la recherche et de l'enseignement supérieur (2010). Formation universitaire au métier d'ingénieur. Repéré à http://www.aeres-evaluation.fr/Actualites/Actualites-de-lagence/ETUDE-Formation-universitaire-au_metier-d-ingenieur.

Aubret, J., Gilbert, P. et Pigeyre, F. (1993). Savoir et pouvoir : les compétences en questions. Paris : P.U.F.

Beaud, S. (2002). 80 \% au bac,... et après ? Les enfants de la démocratisation scolaire. Population, 57, 923-927.

Bédard, D., Turgeon, J., Tardif, J. et Desmarchais, J. (1995). L'apprentissage par problèmes à l'ordre universitaire : fondements, résultats obtenus et limites. Dans Actes du colloque de l'AIPU, Hull, Québec, 8-10 août.

Bédard, D., Lison, C., Dalle, D , Côté, D., et Boutin, N. (2012). Problem-based and Project-based Learning in Engineering and Medicine : Determinants of Students' Engagement and Persistance. Interdisciplinary Journal of Problem-Based Learning, 6(2). http://dx.doi.org/10.7771/1541-5015.1355

Bellier, S. (1999). La compétence. Dans P. Carré et P. Caspar (dir.), Traité des sciences et des techniques de la formation (p. 223-244). Paris : Dunod. 
Berger V. (2012). Assises de l'enseignement supérieur et de la recherche. Rapport au président de la République, 17 décembre. Repéré à http://cache.media.enseignementsup-recherche.gouv.fr/file/ Assises_esr/24/0/Assises-ESR-Rapport-Vincent-Berger-_237240.pdf

Boutin, G. (2004). L'approche par compétences en éducation : un amalgame paradigmatique. Connexions, 81(1), 25-41.

Bruter A. (2008). Le cours magistral comme objet d'histoire. Histoire de l'éducation, (120), 5-32.

Chauvigné, C. et Coulet, J.-C. (2010). L'approche par compétences : un nouveau paradigme pour la pédagogie universitaire? Revue française de pédagogie, 172, 15-28.

Clanet, J. (2001). Etude des organisateurs des pratiques enseignantes à l'université. Revue des Sciences de l'Education, 27(2), 327-352.

Cornu, L. et Vergnioux, A. (1992). La didactique en questions. Paris : CNDP.

Dalle, D., Denis, G., Lachiver, G., Hivon, R., Boutin, N. et Bourque, S. (2002, révision août 2003). L'apprentissage par problèmes et par projets en ingénierie. Nouveaux programmes de génie électrique et de génie informatique. Département de génie électrique et de génie informatique, Université de Sherbrooke. Repéré à https://www.usherbrooke.ca/gelecinfo/fileadmin/sites/gelecinfo/ documents/DOC_Info_APPI.pdf.

De Vecchi, G. et Carmona-Magnaldi, N. (2015). Faire vivre de véritables situations-problèmes. Paris : Hachette éducation.

Derry, S.J., Levin, J.R., Osana, H.P., Jones, M.S. et Peterson, M. (2000). Fostering students'statistical and scientific thinking : Lessons learned from an innovative college course. Am.Educ. Res. J., 37, 747-773.

Dochy, F., Segers, M., Van Den Bossche, P. et Gijbels, D. (2003). Effects of problem-based learning : a meta-analysis. Learning and Instruction, 13(5), 533.

Felouzis, G. (2003). Les mutations actuelles de l'Université (1 $\left.1^{r e} e ́ d.\right)$. Paris : Presses Universitaires de France.

Mollo, V. et Falzon, P. (2004). Auto- and allo-confrontation as tools for reflective activities. Applied Ergonomics, 35(6), 531-540.

Galand, B. et Frenay, M., (2005). L'approche par problèmes et par projets dans l'enseignement supérieur : impact, enjeux et défis. Louvain-la-Neuve : Presses Universitaires de Louvain.

Gerard, F.-M. (2003). L'évaluation de l'efficacité d'une formation. Gestion 2000, 20(3), 13-33. Repéré à www.bief.be.

Hmelo-Silver, C.E. (2004). Problem-based learning : what and how do students learn ? Educational Psychology Review, 16(3), 235-265.

Leplat, J. (2000). La psychologie du travail. Psychologie française, 45(1), 83-96.

Leplat, J. (2004). L'analyse psychologique du travail. Revue Européenne de Psychologie Appliquée/ European Review of Applied Psychology, 54(2), 101-108.

Newman, M. J. (2005). Problem-based learning : an introduction and overview of the key features of the approach.J Vet Med Educ, 32(1), 12-20. Repéré à http://www.utpjournals.com/jvme/tocs/ 321/12.pdf.

Pastirik, P. J. (2006). Using problem-based learning in a large classroom. Nurse Education in Practice, 6, 261-267. 
Peretti, C. (dir.) (2009). Rapport sur les Centres d'Initiation à l'Enseignement Supérieur ( $\mathrm{n}^{\circ}$ 2009-055).

Perrenoud, P. (1997). La pédagogie différenciée. Paris : ESF.

Raucent, B. et Milgrom, E. (2011). Guide pratique pour une pédagogie active : les APP, Apprentissages par Problèmes et par Projets. Toulouse : INSA Toulouse.

Rey, B., Carette, V., Defrance, A. et Kahn, S. (2012). Les compétences à l'école : apprentissage et

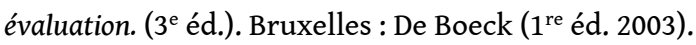

Ria, L. (2009). De l'analyse de l'activité des enseignants débutants en milieu difficile à la conception de dispositifs de formation. Dans M. Durand et L. Filliettaz (éd.), Travail et Formation des Adultes (p. 217-243). Paris : PUF.

Roegiers, X. (2014). Quelle évaluation des compétences, au service de quel projet pour l'école? Dans B. Rey, C. Dierendonck et E. Loarer, L'évaluation des compétences en milieu scolaire et en milieu professionnel (p. 73-111). Bruxelles : De Boeck.

Romainville, M. (2000). L'échec dans l'université de masse. Paris : L'Harmattan.

Savin-Baden, M. (2000). Problem-Based Learning In Higher Education : Untold Stories. Londre : McGraw-Hill Education.

Tardif, J. (2003). Développer un programme par compétences : de l'intention à la mise en œuvre. Profesorado. Revista de Currículum y Formación del Profesorado, 12(3), 16.

Vernon, D.T.A. et Blake, R.L. (1993). Does problem-based learning work? A meta-analysis of evaluative research. Academic Medicine, 68, 550-563.

\section{NOTES}

1. Téléchargeable sur le site du réseau FIGuRe: http://reseau-figure.fr/le-reseau-figure/ documents-de-reference/

2. Gain relatif énoncé par Gérard (2003): $G R=$ [score(post-test) - score (pré-test)]/ [score (maximum possible) - score(pré-test)].

\section{RÉSUMÉS}

Face à un public massif et divers d'étudiants, la question de la transmission de connaissances et de l'évolution des pratiques pédagogiques dans l'enseignement supérieur est actuellement au cœur des débats. Nous assistons au sein des universités au développement d'une variété d'expérimentations pédagogiques (Clanet, 2001), remettant ainsi en question la pertinence du cours magistral. Pourtant, un certain nombre d'enseignants continuent à enseigner de manière dite «traditionnelle ». La question de l'efficacité de ces implantations pédagogiques par rapport à un cours traditionnel se pose donc toujours. Notre étude s'est intéressée à étudier l'efficacité d'un apprentissage par problèmes par rapport à un apprentissage traditionnel, dans un cursus Master ingénierie sur l'unité d'enseignement Étude des systèmes mécaniques. Nos résultats tendent à montrer que le dispositif d'apprentissage par problèmes est efficace, et ceci 
rapidement pour des étudiants qui n'ont pas l'habitude d'être actifs. La mise en activité, la responsabilisation et l'engagement des étudiants sont certainement des facteurs de réussite à explorer.

Faced with a massive influx of students without a common educational foundation and background, the issue of knowledge transfer and of the development of teaching practices in higher education is currently at the crux of the debate. We assist in the development of a variety of educational experiments within universities (Clanet, 2001), thus calling into question the relevance of lectures. Yet, a number of professors continue to teach the "traditional" way. The question of the effectiveness of these educational implementations compared to a traditional class consequently still arise. Our study is interested in measuring and comparing the effectiveness of a Problem-based Learning (PBL) compared to a traditional education in a Program Master's degree Engineering on the unit of education: Study of Mechanical Systems. Our results suggest that the Problem-based learning are effective, and this rapidly for students who are not used to active learning. Starting students going, responsibility awareness, and students' commitment are certainly factors of success to explore.

INDEX

Mots-clés : apprentissage par problèmes (APP), efficacité, compétences, pédagogie universitaire

\section{AUTEURS}

PERRINE MARTIN

Maître de conférences, ADEF EA 4671, Aix Marseille Université

\section{PHILIPPE PADULA}

ADEF EA 4671, Aix Marseille Université 